\title{
Effects of Contact with Stillborn Babies on Maternal Anxiety and Depression
}

\author{
Joanne Cacciatore, $P h D, M S W, F T$, Ingela Rådestad, $P h D$, and J. Frederik Frøen, $M D, P h D$
}

\begin{abstract}
Background: Some guidelines encourage mothers to see and hold their babies after stillbirth, which might be traumatizing. The study objective was to investigate the effects of women seeing and holding their stillborn baby on the risk of anxiety and depression in a subsequent pregnancy and in the long term. Methods: Thirty-seven organizations recruited women who had experienced stillbirth ( $\mathrm{N}=2,292$ of whom 286 reported being pregnant). Anxiety and depressive symptoms were assessed by using the 25-item Hopkins Symptom Check List. Results: Among nonpregnant women, seeing and holding their stillborn baby were associated with lower anxiety symptoms (OR 0.68, 95\% CI 0.49-0.95) and a tendency toward fewer symptoms of depression (OR 0.72, 95\% CI 0.51-1.02), compared with pregnant women. Participants who were pregnant also had less depressive symptomatology (OR 0.57, 95\% CI 0.43-0.75), but more symptoms of anxiety if they had seen and held their baby (OR 3.79, 95\% CI 1.42-10.1). Conclusions: Seeing and holding the baby are associated with fewer anxiety and depressive symptoms among mothers of stillborn babies than not doing so, although this beneficial effect may be temporarily reversed during a subsequent pregnancy. (BIRTH 35:4 December 2008)
\end{abstract}

Key words: stillbirth, fetal death, psychosocial care, anxiety, depression

During the mid-20th century, when both birth and death were institutionalized in the Western world, hospitals took over the management of stillbirth. Mothers rarely had an opportunity to see, hold, or take photographs of their stillborn babies. Instead, "babies were whisked away and disposed of" to minimize the psychological trauma of the experience (1). However, in the late-1970s, physicians, such as Emmanuel Lewis, began to criticize the "rugby pass management of stillbirth ... the catching of a stillbirth after delivery, the quick accurate back-pass through the labor room door to someone who catches the baby" (1).
In 1983, Lovell reported on 22 mothers of whom 12 never saw or held their stillborn babies (2). She found that women "usually relied on the judgment of the health professionals ... and took her cues from the experts." Women trusted those in authority as to whether or not they should see or hold their stillborn babies. Their narratives showed that they regretted not having contact with their babies, and these mothers had difficulty reconciling: "Sandy wonders what happened to her stillborn son: 'I have a recurring dream that I'm in the hospital searching for him. I feel it is the most important event in my life. And yet, it is a terrible nothingness"” (2, p 758).
Joanne Cacciatore is an Assistant Professor in the College of Human Services at Arizona State University, Phoenix, Arizona, USA; Ingela Radestad is an Associate Professor in the Department of Caring Sciences and Public Health at Mälardalens University, Eskilstuna, Sweden; and J. Frederik Frøen is a Director and an Associate Professor in the Department of Genes and Environment, Division of Epidemiology at the Norwegian Institute of Public Health, Oslo, Norway.

The study received financial support from the Norwegian Society for Unexpected Infant Death, Oslo, Norway.
Address correspondence to Joanne Cacciatore, College of Human Services, Arizona State University, 4701 West Thunderbird Road, Glendale, Arizona 85308, USA.

Accepted June 5, 2008

(C) 2008, Copyright the Authors

Journal compilation (c) 2008, Wiley Periodicals, Inc. 
In response to criticism by some physicians, nurses, and grieving mothers, many hospitals began to promote rituals through which mothers could see and hold their stillborn babies, believing this process would facilitate healthy maternal grief responses (3). Staff stopped acting as if stillbirth was a nonevent, and caregivers began to encourage mothers to meet and say farewell to their babies $(4,5)$. It was an important change in policy (6-8). DeFrain et al studied 350 families experiencing stillbirth, concluding that seeing and remembering the stillborn baby "is essential to the vast majority" (3). In a study of 314 women in a population-based setting, Rådestad et al found improved long-term outcomes for mothers who have had contact with their stillborn babies compared with mothers who did not have such contact (4).

However, more recently, this protocol has been called into question. In a study of 53 mothers with a previous pregnancy ending in a stillbirth, Hughes et al reported a higher risk of disorganization in children of mothers who had experiences of seeing and holding their stillborn baby compared with mothers who had no such experiences (9). In another study, the same research group examined 65 women during a subsequent pregnancy after a stillbirth and reported that behaviors that promoted contact with the stillborn baby were associated with a worse outcome (10). These findings suggest that, rather than facilitating healthy grieving, seeing and holding the stillborn baby are correlated with depression and anxiety in bereaved mothers.

The level of contact between a mother and her stillborn is not amenable to controlled trials and the existing evidence to guide care is scant. Until the studies by Hughes et al were published, the development of care protocols was driven by the consensus of observational studies. Whether the results of Hughes et al truly represent a discrepancy from earlier studies, are confounded by small numbers and lacking covariates, or only describe a transient effect in a subsequent pregnancy remains an important question for future improvements of care.

The aim of this study was to determine the associations of seeing and holding the stillborn baby with maternal anxiety and depression using the 25-item Hopkins Symptom Check List (HSCL) to estimate the main outcomes. We assessed factors affecting both the mothers' probability of seeing and holding their babies and the risk for symptoms of anxiety and depression during a subsequent pregnancy and in the long term.

\section{Methods}

We searched 12 Internet search engines and directories for the terms "stillbirth," "pregnancy loss," "fetal loss," or "fetal death." Where possible, we included all inflections of the terms and we updated the past 3 months only in pages in English. From the first 100 matches from each search engine, 749 Web pages were identified, which were examined to identify organizations and Web sites offering free information on pregnancy and childbirth (including stillbirth) or information, support, or Web forums for families affected by stillbirth. We excluded sites presenting only sporadic information as, for example, online scientific journals, dictionaries or databases, and sites for scientific, professional, or governmental use, such as the American College of Obstetricians and Gynecologists or U.S. National Center for Health Statistics. The 104 sites or organizations were invited to recruit women affected by stillbirth to respond to a questionnaire through their Web sites, mailing lists, newsletters, forums, or other means using ready-made invitations. Thirty-seven organizations accepted.

The study Maternal Observations and Memories of Stillbirth (MOMStudy) was established, and the Web site http://www.momstudy.com, containing the questionnaire was open in the period from February 8, 2004, to September 15, 2005. We monitored entries by a single Internet protocol address using The Webalizer $^{\odot}$, freeware from http://www.mrunix.net.

In all, 3,519 women reported a stillbirth. Among them, 2,900 participants completed the entire questionnaire and confirmed their submission (completion rate $82.4 \%$ ). These stillbirths included 2,769 singletons, 119 twins, and 12 triplets. We included the 2,292 women who reported a singleton stillbirth after 20 weeks' gestation and certainty about whether or not they were currently pregnant $(n=286)$. Participants were mainly recruited from the United States (72\%), United Kingdom (11\%), Australia (9\%), and Canada (5\%). Among the stillborns were 52.2 percent boys and 47.8 percent girls.

The questionnaire was interactive to minimize the number of nonapplicable questions to each participant. The questionnaire explored various topics related to stillbirths, including also indirect repeat questions to confirm accuracy (e.g., asking the participant's current age and later asking her age when experiencing stillbirth and years since it happened). Cases with inconsistencies were reviewed for extreme values, but only one report was excluded due to inconsistencies that could not be explained by obvious unintended errors.

The study protocol was approved by the Regional Ethics Committee for medical research of Southern Norway.

\section{Data Analysis}

Anxiety- and depression-related symptoms were measured at the beginning of the questionnaire by the 
25-item HSCL (11). We defined an average symptom score for anxiety symptoms greater than 1.75 as anxiety and likewise for depression (11) and used these scores as dichotomous dependent variables for logistic regressions of the main outcomes presented in Tables 3 and 4 .

We used SPSS 14.0 for all statistical analyses (12). Maternal characteristics are presented in Table 2, and all potentially confounding factors (associated with both the dependent and the independent variable) with $p<0.2$ were introduced in multivariable stepwise backward logistic regressions in all further analyses. All covariates were entered in the regressions as continuous, dichotomous, or categorical data. Participant's pregnancy status while responding, marital status, and mode of recruitment had no confounding effects. Pregnancy status was used for stratification and marital status was excluded, whereas mode of recruitment was introduced in subsequent analyses despite the lack of confounding effect. No final analysis of nonpregnant $(n=2,006)$ or pregnant $(n=$ 286) women included more than 6 or 3 covariates, respectively.

\section{Results}

The great majority of the mothers saw (95\%) and held $(90 \%)$ their stillborn babies and very few expressed regret. However, almost 80 percent of those who did not see and hold the baby regretted that they did not do so (Table 1). The main reason for the lack of contact with the baby was maternal choice; but for dressing or washing the baby, 84.3 percent did not perceive that as an option. Few women felt any pressure to have contact or to avoid contact with their babies. Among mothers who did not see their babies, 9.5 percent had felt pressured to do so; conversely, 10.3 percent had felt pressured not to do so.

Fewer mothers saw or held their babies if they were born with congenital anomalies compared with mothers whose babies died of other causes (OR 0.37, $95 \%$ CI $0.20-0.69)$. Congenital malformations, gender, and living in the U.S. or other countries had no effect on any of the analyses presented here and were excluded as covariates. The characteristics of participants are presented in Table 2 and only two of these factors differed significantly among those exposed to their stillborn or not: mothers who saw and held their stillborn experienced a third trimester loss more often ( $81 \mathrm{vs} 66 \%, p<0.001$ ) and they also experienced their loss 1.09 years later $(p<0.001)$ than those who did not (Table 3).

Among participants, 42 and 62 percent reported symptoms of anxiety and depression, respectively.

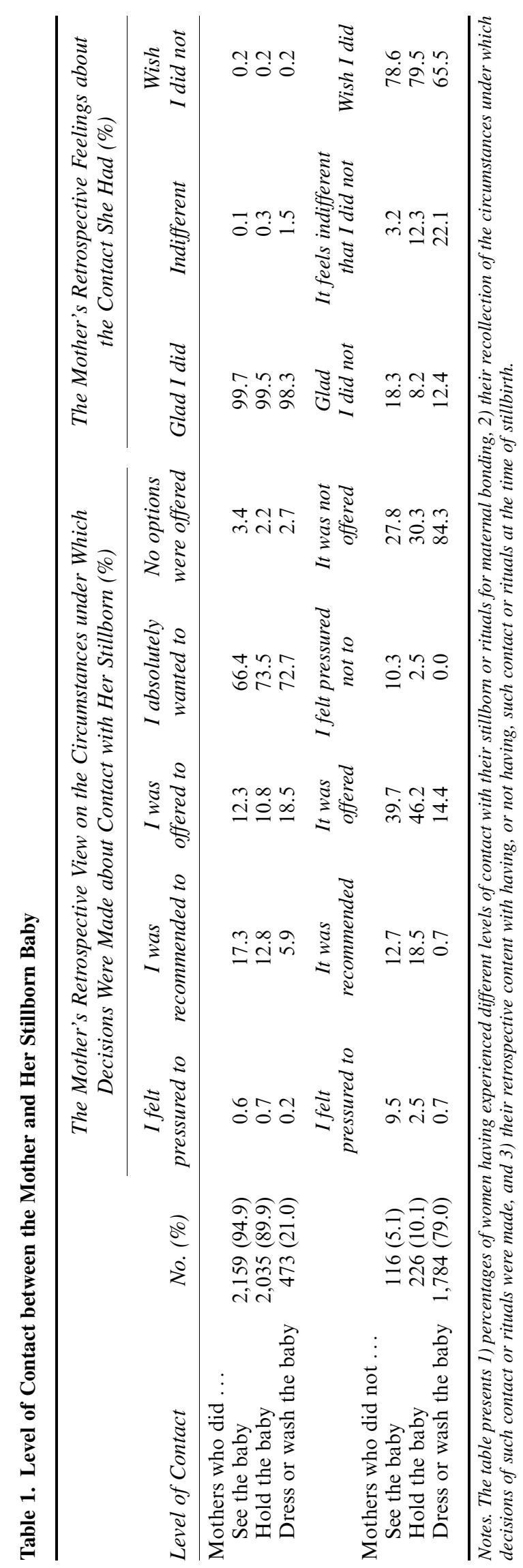




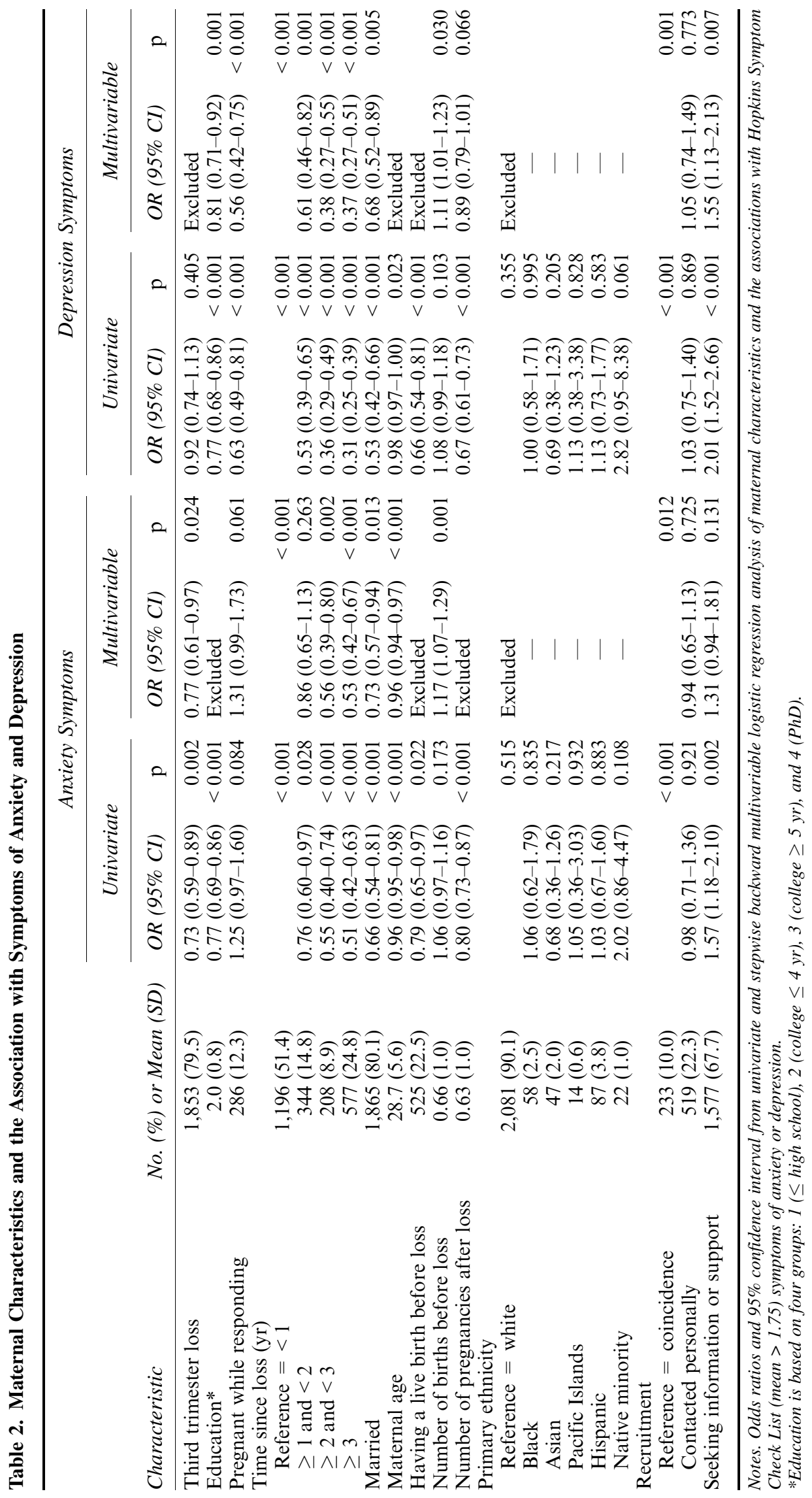


Table 3. Maternal Characteristics and the Association with Whether or Not the Mother Held Her Baby

\begin{tabular}{|c|c|c|c|c|c|c|}
\hline \multirow[b]{2}{*}{ Characteristic } & \multirow{2}{*}{$\begin{array}{l}\text { Did Not Hold } \\
\text { Her Stillborn } \\
\text { No. }(\%) \text { or } \\
\text { Mean (SD) }\end{array}$} & \multirow{2}{*}{$\begin{array}{c}\text { Held Her Stillborn } \\
\text { No. }(\%) \text { or } \\
\text { Mean (SD) }\end{array}$} & \multicolumn{2}{|c|}{ Univariate } & \multicolumn{2}{|c|}{ Multivariable } \\
\hline & & & $\begin{array}{c}\text { OR } \\
(95 \% C I)\end{array}$ & $\mathrm{p}$ & $\begin{array}{c}O R \\
(95 \% C I)\end{array}$ & $\mathrm{p}$ \\
\hline Third trimester loss & $150(66.4)$ & $1,651(81.1)$ & $2.18(1.62-2.93)$ & $<0.001$ & $2.34(1.73-3.16)$ & $<0.001$ \\
\hline Education* & $2.0(0.8)$ & $2.1(0.8)$ & $1.15(0.96-1.39)$ & 0.133 & Excluded & \\
\hline Pregnant while responding & $25(11.1)$ & $249(12.2)$ & $1.12(0.73-1.73)$ & 0.608 & Excluded & \\
\hline \multicolumn{7}{|l|}{ Time since loss (yr) } \\
\hline Reference $=<1$ & 87 (49.7) & $746(47.8)$ & & 0.014 & & 0.003 \\
\hline$\geq 1$ and $<2$ & $22(12.6)$ & $268(17.2)$ & $1.18(0.77-1.81)$ & 0.440 & $1.17(0.76-1.79)$ & 0.486 \\
\hline$\geq 2$ and $<3$ & $8(4.6)$ & $163(10.4)$ & $2.11(1.09-4.10)$ & 0.028 & $2.24(1.15-4.37)$ & 0.018 \\
\hline$\geq 3$ & $58(33.1)$ & $385(24.6)$ & $0.76(0.55-1.04)$ & 0.084 & $0.70(0.51-0.96)$ & 0.029 \\
\hline Married & $182(80.5)$ & $1,638(80.6)$ & $1.00(0.71-1.42)$ & 0.989 & Excluded & \\
\hline Maternal age & $27.8(5.8)$ & $28.7(5.5)$ & $1.03(1.01-1.06)$ & 0.13 & Excluded & \\
\hline Having a live birth before loss & $41(18.1)$ & $463(22.8)$ & $1.33(0.93-1.89)$ & 0.114 & Excluded & \\
\hline Number of births before loss & $0.59(1.0)$ & $0.66(1.0)$ & $1.07(0.92-1.25)$ & 0.363 & Excluded & \\
\hline Number of pregnancies after loss & $0.66(1.1)$ & $0.62(1.0)$ & $0.96(0.85-1.10)$ & 0.582 & Excluded & \\
\hline \multicolumn{7}{|l|}{ Primary ethnicity } \\
\hline Reference $=$ white & $197(87.9)$ & $1,827(90.5)$ & & 0.253 & Excluded & \\
\hline Black & $5(2.2)$ & $51(2.5)$ & $1.10(0.43-2.79)$ & 0.841 & - & \\
\hline Asian & $9(4.0)$ & $35(1.7)$ & $0.42(0.20-0.89)$ & 0.023 & - & \\
\hline Pacific Islands & $2(0.9)$ & $10(0.5)$ & $0.54(0.12-2.47)$ & 0.427 & - & \\
\hline Hispanic & $11(4.9)$ & $74(3.7)$ & $0.73(0.38-1.39)$ & 0.725 & - & \\
\hline Native minority & $0(0.0)$ & $21(1.0)$ & - & - & - & \\
\hline \multicolumn{7}{|l|}{ Recruitment } \\
\hline Reference $=$ coincidence & $42(10.6)$ & $205(10.1)$ & & 0.564 & Excluded & \\
\hline Contacted personally & $44(19.5)$ & $459(22.6)$ & $1.22(0.72-2.06)$ & 0.455 & & \\
\hline $\begin{array}{l}\text { Seeking information } \\
\text { or support }\end{array}$ & $158(69.9)$ & $1,368(67.3)$ & $1.01(0.64-1.60)$ & 0.953 & & \\
\hline
\end{tabular}

Notes. Odds ratios and 95\% confidence interval from univariate and stepwise backward multivariable logistic regression analysis of the associations of maternal characteristics with whether she held her stillborn or not.

*Education is based on four groups: 1 ( $\leq$ high school), 2 (college $\leq 4$ yr), 3 (college $\geq 5$ yr), and 4 (PhD).

Table 4. Maternal Symptoms and the Association with the Conditions under Which the Mother Decided to See Her Stillborn Child

\begin{tabular}{|c|c|c|c|c|c|c|}
\hline \multirow{2}{*}{$\begin{array}{l}\text { Contact with } \\
\text { Baby or Not }\end{array}$} & \multirow{2}{*}{$\begin{array}{c}\text { Conditions under Which Mother } \\
\text { Had Contact with Baby }\end{array}$} & \multirow[b]{2}{*}{$\%$} & \multicolumn{2}{|c|}{ Anxiety Symptoms } & \multicolumn{2}{|c|}{ Depression Symptoms } \\
\hline & & & OR $(95 \% C I)$ & $\mathrm{p}$ & OR $(95 \% C I)$ & $\mathrm{p}$ \\
\hline \multirow{4}{*}{$\begin{array}{l}\text { Did not see her } \\
\quad \text { stillborn }(n=116)\end{array}$} & Conditions (reference $=$ own wish) & 10.3 & & 0.215 & & 0.786 \\
\hline & Felt pressured to see the stillborn & 9.5 & $2.05(0.29-14.6)$ & 0.476 & $0.80(0.10-6.64)$ & 0.832 \\
\hline & Was not given any option & 27.8 & $4.93(0.89-27.4)$. & 0.068 & $0.62(0.11-3.53)$ & 0.588 \\
\hline & $\begin{array}{l}\text { Was offered to see the } \\
\text { stillborn as an option }\end{array}$ & 39.7 & $2.17(0.42-11.3)$ & 0.358 & $0.75(0.14-4.16)$ & 0.745 \\
\hline \multirow{6}{*}{$\begin{array}{l}\text { Saw her stillborn } \\
\quad(n=2,129)\end{array}$} & $\begin{array}{l}\text { Was recommended to } \\
\text { see the stillborn }\end{array}$ & 12.7 & $5.69(0.80-40.3)$ & 0.082 & $1.99(0.24-16.8)$ & 0.526 \\
\hline & Conditions (reference $=$ own wish) & 66.4 & & 0.684 & & 0.019 \\
\hline & Felt pressured to see the stillborn & 0.6 & $2.22(0.74-6.69)$ & 0.156 & $2.29(0.67-7.84)$ & 0.187 \\
\hline & Was not given any option & 3.4 & $0.97(0.58-1.62)$ & 0.909 & $0.58(0.35-0.96)$ & 0.035 \\
\hline & $\begin{array}{l}\text { Was offered to see the } \\
\text { stillborn as an option }\end{array}$ & 12.3 & $1.04(0.78-1.39)$ & 0.790 & $1.40(1.03-1.91)$ & 0.033 \\
\hline & $\begin{array}{l}\text { Was recommended to } \\
\text { see the stillborn }\end{array}$ & 17.3 & $0.96(0.74-1.24)$ & 0.733 & $0.97(0.75-1.25)$ & 0.783 \\
\hline
\end{tabular}

Notes. Adjusted odds ratios and 95\% confidence interval from multivariable logistic regression analysis of the associations of maternal symptoms with the conditions under which women made the decision to see or not see their stillborn after delivery. 
Maternal age and the amount of time since the stillbirth were associated with fewer symptoms of anxiety; that is, younger mothers and mothers with a recent loss had more anxiety symptoms. In addition, third trimester losses (vs second trimester losses), being married (vs unmarried or divorced/widowed), low parity at the time of loss (vs higher parity), and having experienced births after their loss (vs not having experienced births) were associated with fewer symptoms of anxiety (Table 2). Likewise, maternal education level and time since stillbirth were associated with fewer symptoms of depression (i.e., mothers with little education and a recent loss had more depression symptoms). In addition, previous live births (vs none), being married (vs single), low parity at the time of loss (vs higher parity), and having experienced births after their loss (vs not having experienced births) were associated with lower levels of depressive symptoms.

Women recruited to the study while actively seeking support or information had more recent stillbirths and thus more symptoms than women not actively seeking support. Being pregnant after a loss was associated with fewer depressive symptoms and a tendency toward higher anxiety symptomatology compared with not being pregnant at the time of the study (Table 2).

Maternal characteristics were not associated with whether a woman held her stillborn baby or not, but those who experienced third trimester stillbirths more often held their baby, and the frequency in holding differed with the number of years since the stillbirth occurred (Table 3). Among women who were not pregnant when responding to the questionnaire, seeing the baby was associated with lower levels of anxiety and depression (table with data available from authors on request). Compared with the significant step of seeing the baby, each successive degree of contact did not add significantly to the effect. For pregnant women, the effects were reversed with increased risk of anxiety if they had held the stillborn baby and a tendency toward depression.

Among mothers who did not see their stillborn babies, those who never were given the opportunity and those who actively resisted tended to have more anxious symptoms than mothers who simply reported that they did not wish to see the baby (Table 4). Among mothers who saw their stillborn babies, those who reported that they were given no other options or that it was not a subject that was discussed with them had fewer symptoms of depression than mothers who reported to have seen the baby because of their own wish to do so. Conversely, mothers who perceived that health care practitioners placed the responsibility of decision making on them by offering them the option to see and hold their baby when they had no strong wishes of their own tended to have more symptoms of depression than those who spontaneously wished to see their baby. In terms of the woman's retrospective regret over seeing or not seeing her baby, the conditions under which she saw or held her baby had no impact on regret if the mother actually had the contact described. However, those who did not have the contact had more regrets if not offered this as an option, in particular dressing or washing the baby $(p<0.001)$, compared with women who did have such contact.

\section{Discussion}

Ninety-five percent of all mothers in this study saw and 90 percent held their stillborn baby and nearly all appreciated the moments they had with their babies. Seeing and holding their baby were associated with fewer symptoms of anxiety and depression than not seeing and holding their baby. Most mothers who did not see and hold their babies regret lost opportunities of contact.

Our main findings of a beneficial, long-term effect for mothers having had contact with their stillborn babies support findings from previous studies $(8,13)$. Missed opportunities for contact with the baby were associated with retrospective regret in this study. The caregivers' demeanor at the time of stillbirth was associated with long-term outcomes. Mothers had fewer depressive symptoms if they perceived that seeing the baby was not simply an option they could choose compared with mothers who spontaneously wished to see their baby. Thus, messages conveyed by the staff through both verbal and nonverbal communication may influence and shape the mother's perceptions of expected, normal responses. If caregivers convey the attitude that seeing and holding the baby are equally as normal after a stillbirth as after a live birth, mothers respond positively. Conversely, the attitude that seeing a stillborn baby is an option the mother must choose, implying that this behavior is not necessarily normal or acceptable, may strengthen the sense of abnormality in the situation.

Among mothers currently pregnant at the time of investigation, the findings in this study were reversed, confirming previous reports that seeing and holding the stillborn baby are associated with more hardships during future pregnancies (10). Yet, the long-term results among those who were not pregnant at the time of the study, compared with those who were pregnant at the time or who had not had a subsequent birth after a loss and the fact that having experienced births after a loss was associated with lower levels of both anxiety and depression, suggest that these negative effects are transient. Rather than discouraging contact with the stillborn baby, additional support 
should be offered during subsequent pregnancies after a loss.

Hospital bereavement teams often manage perinatal death programs that support contact with a dead or dying newborn $(1,5)$. Lasker and Toedter found that "parents who experience interventions such as having a photograph or keepsake were significantly more satisfied" than those having not participated in such ritualistic acts $(1, \mathrm{p} 41)$. In an observational study, long-term anxiety was lower among women having tokens of remembrance from their baby than among women not having them (8). Rituals, such as holding, caressing, and caring for the dead body of a loved one, have been used universally by humans throughout history and across cultures as a way of realizing and coping with loss $(14,15)$ and they have been described as the antidote to powerlessness (16). Rituals transform and mediate grief, facilitating a symbolic connection to the deceased (17). This type of contact begets memories that stillbirth families would not otherwise have an opportunity to create (18).

This study has the advantage of a very large number of participants needed to enable the inclusion of significant confounders in the analyses. However, despite a broad-based approach for recruitment, bias may have been introduced not only by depending on informed consent from participants but also by a skewed recruitment of participants in comparison with a total population. Participants represent younger and higher socioeconomic groups (Internet access), and the adjustments for age and educational level in our analyses will not fully correct this fact. A large proportion of women were recruited while actively searching for support or information about stillbirths on the Internet. As seen in our results, these women had more symptoms than those who were actively contacted by participating organizations. However, no associations were found between the mode of recruitment and the prevalence of seeing and holding the child (Table 3). Despite not being a probable confounder, the mode of recruitment was included as a categorical covariate in all multivariable analyses.

Participants' self-selection into the study may have been correlated with character traits that may influence the woman's psychological response to having a stillborn baby. Methodologically, the selection effect from self-selected participants may compromise the generalizability of the outcomes. In addition, a portion of the study focuses on the perceptual experiences of women while interacting with caregivers. The potential for recall bias may arise in the rating of the experiences with caregivers, particularly for women who may have acute depressive disorders that predated the stillbirth; the potential also exists that caregivers identified the less anxious mothers, and thus, they may have been more likely to include them in decision making around holding and seeing the baby who died.

The use of parental support groups for recruitment may introduce bias by recruiting stillbirth parents with fixed views. As the findings of negative effects of seeing and holding the child caused outrage and disbelief in much of the stillbirth parent community, any possible bias would tend to overestimate our findings of positive effects of contact and of regret for missed opportunities. However, the MOMStudy addressed many aspects of stillbirth, and no mention of the fact that these questions would be addressed was included in any recruitment material for the study. Rather, the recruitment materials focused on gaining knowledge for the prevention of stillbirths. In addition to no effect of mode of recruitment on level of contact, participants recruited through parental groups had symptoms identical to those who found the study "by coincidence" (Table 2). The fact that this study confirms previous reports of negative effects of contact during subsequent pregnancies contradicts the existence of such bias of significance.

\section{Conclusions}

The psychosocial effects of stillbirth are long lasting and traumatic, leaving those in its wake more vulnerable to anxiety and depression $(3,19-21)$. Women in this study overwhelmingly wanted to see and hold their babies and very few expressed regret in doing so. Seeing and holding a stillborn baby are associated with fewer anxiety and depressive symptoms among mothers of stillborn babies than not doing so, although this beneficial effect may be temporarily reversed during a subsequent pregnancy. Thus, mothers should be offered additional support during a subsequent pregnancy rather than discouraged from seeing and holding their stillborn baby. Since caregiver interaction may influence a grieving mother's responses during the acute crisis, active management that includes responsiveness, support, and a staff willing to facilitate contact with a stillborn baby is necessary and may provide long-term benefits to the mother.

\section{Acknowledgments}

We thank MISS Foundation, the National Stillbirth Society, all other recruiting organizations, and all participating mothers for their efforts in this study. We gratefully acknowledge Moni Ryczek, Jon Martin Solaas, and Mary Geitz and Gary McSpadden for coordinating recruitment, development of the interactive questionnaire, and hosting the Web site, respectively. 


\section{References}

1. Lasker JN, Toedter LJ. Satisfaction with hospital care and interventions after pregnancy loss. Death Stud 1994;18(1): 41-64.

2. Lovell A. Some questions of identity: Late miscarriage, stillbirth, and perinatal loss. Soc Sci Med 1983;17(11): 755-761.

3. DeFrain J, Martens L, Stork J, Stork W. The psychological effects of a stillbirth on surviving family members. Omega $J$ Death Dying 1990;22(2):81-108.

4. Rådestad I, Nordin C, Steineck G, Sjogren B. Stillbirth is no longer managed as a nonevent: A nationwide study in Sweden. Birth 1996;23:209-215.

5. Kozick P, Brown CE. Reconstructing reality: Preserving memories through the creation of impressions. Int J Childbirth Educ 1994;9(11):38.

6. Sadler ME. When your patient's baby dies before birth. $R N$ 1987;50(8):28-31.

7. Zeanah $\mathrm{CH}$, Harmon RJ. Perinatal loss and infant mental health: An introduction. Infant Mental Health J 1995;16(2): 76-93.

8. Rådestad I, Steineck G, Nordin C, Sjögren B. Psychological complications after stillbirth-Influence of memories and immediate management: Population based study. BMJ 1996; 312(7045):1505-1508.

9. Hughes P, Turton P, Hopper E, Evans CDH. Assessment of guidelines for good practice in psychosocial care of mothers after stillbirth: A cohort study. Lancet 2002;360(9327): 114-118.
10. Hughes P, Turton P, Hopper E, McGauley GA. Disorganized attachment behavior among infants born subsequent to stillbirth. J Child Psychiatr 2001;42(6):791-801.

11. Derogatis LR, Lipman RS, Rickels K, et al. The Hopkins Symptom Checklist (HSCL): A self-report symptom inventory. Behav Sci 1974;19:1-15.

12. SPSS. Statistical Package for the Social Sciences. Version 14.0.2. Chicago, Illinois: SPSS Inc., 2003.

13. Trulsson O, Rådestad I. The silent child-Mothers' experiences before, during, and after stillbirth. Birth 2004;31(3): 189-195.

14. Beder J. Mourning the unfound: How we can help. Fam Soc 2002;83(4):400-403.

15. Boss P. Ambiguous Loss. Cambridge, Massachusetts: Harvard University Press, 1990.

16. Miller S. Finding Hope When a Child Dies: What Other Cultures Can Teach Us. New York: Simon and Schuster, 1999.

17. Romanoff BD, Terenzio M. Rituals and the grieving process. Death Stud 1998;22:697-711.

18. Vaisanen L. Family Grief and Recovery Process When a Baby Dies. Finland: Department of Psychiatry, University of Oulu, Oulu, 1999.

19. Goldenberg RL, Kirby R, Culhave JF. Stillbirth: A review. J Matern Fetal Neonatal Med 2004;16:79-95.

20. Kirkley-Best E, Kellner KR. The forgotten grief: A review of the psychology of stillbirth. Am J Orthopsychiatry 1982;52(3): 420-429.

21. Cacciatore J. Effects of support group participation on post traumatic stress responses in women experiencing stillbirth. Omega J Death Dying 2007;55(1):71-90. 